\title{
First Case of Esophagectomy Using a Robotic Single-Port System for Laryngo-Esophagectomy
}

\author{
Seong Yong Park, M.D., Ph.D. \\ Department of Thoracic and Cardiovascular Surgery, Yonsei University College of Medicine, Seoul, Korea
}

\section{ARTICLE INFO}

Received September 8, 2021

Revised November 11, 2021

Accepted November 16, 2021

Corresponding author

Seong Yong Park

Tel $82-2-2228-2141$

Fax 82-2-2228-2140

E-mail syparkcs@gmail.com

ORCID

https://orcid.org/0000-0002-5180-3853

${ }^{\dagger}$ Current affiliation: The author is now with the Department of Thoracic and Cardiovascular Surgery, Samsung Medical Center, Sungkyunkwan University School of Medicine, Seoul, Korea.

\begin{abstract}
A 58-year-old female patient was diagnosed with hypopharyngeal cancer with extension to the invasion of the upper esophagus. After 2 cycles of durvalumab as neoadjuvant therapy, total laryngo-esophagectomy using a single-port (SP) system via a subcostal incision was done. The operation was completed within 41 minutes, and the patient recovered without esophagectomy-related complications. The patient received total laryngectomy and esophagectomy using a robotic SP system via a 3-cm-long subcostal incision and gastric pull-up under laparotomy. During the postoperative period, the patient suffered from anastomotic leakage, but recovered with vacuum therapy. Here, we report the first successful human case of esophagectomy using an SP system.
\end{abstract}

Keywords: Esophagectomy, Robotic surgical procedure, Single port surgery, Case report

\section{Case report}

A 58-year-old female patient was referred from a local hospital due to dysphagia. Neck computed tomography and magnetic resonance imaging revealed a $4.5-\mathrm{cm}$ longitudinal mass that invaded the hypopharynx and cervical esophagus, extending to the upper esophagus (Fig. 1A, B). Biopsy revealed that the mass was moderately differentiat- ed squamous cell carcinoma. After 2 cycles of durvalumab as neoadjuvant therapy, total laryngo-esophagectomy with neck dissection and gastric pull-up was planned.

The patient was placed in a semi-prone position after double-lumen intubation. A 5 -mm assistant port was inserted in the posterior axillary line in the eighth intercostal space to insufflate the chest cavity with $12 \mathrm{~mm} \mathrm{Hg}$ carbon dioxide and to displace the diaphragm inferiorly. A skin
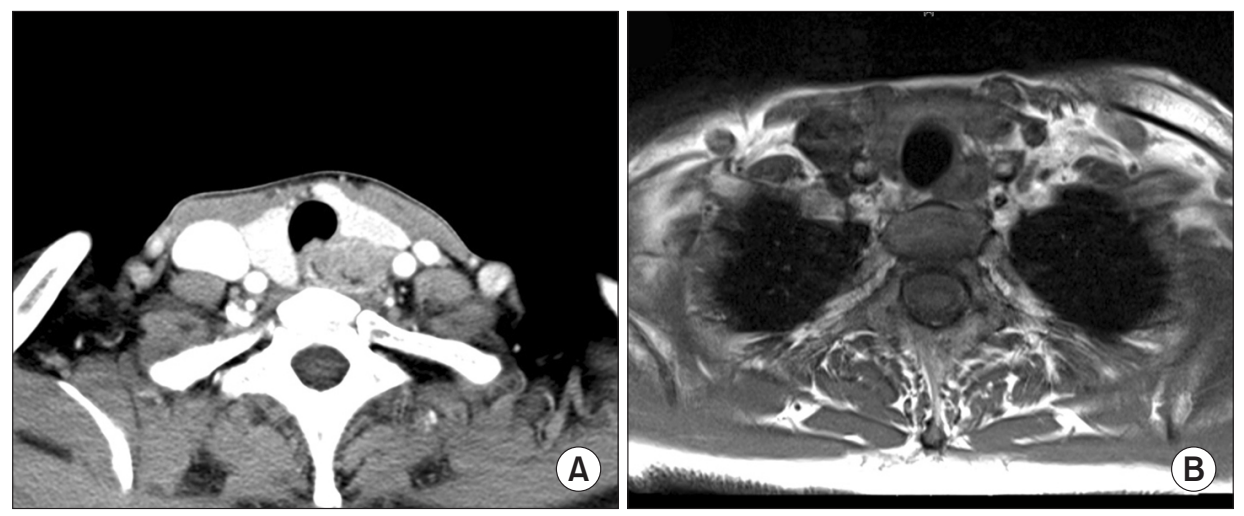

Fig. 1. (A) Initial neck computed tomography of the patient. (B) Initial neck magnetic resonance image of the patient.

Copyright (C) 2022, The Korean Society for Thoracic and Cardiovascular Surgery

(c) (i) This is an Open Access article distributed under the terms of the Creative Commons Attribution Non-Commercial License (http://creativecommons.org/licenses/ by-nc/4.0) which permits unrestricted non-commercial use, distribution, and reproduction in any medium, provided the original work is properly cited. 

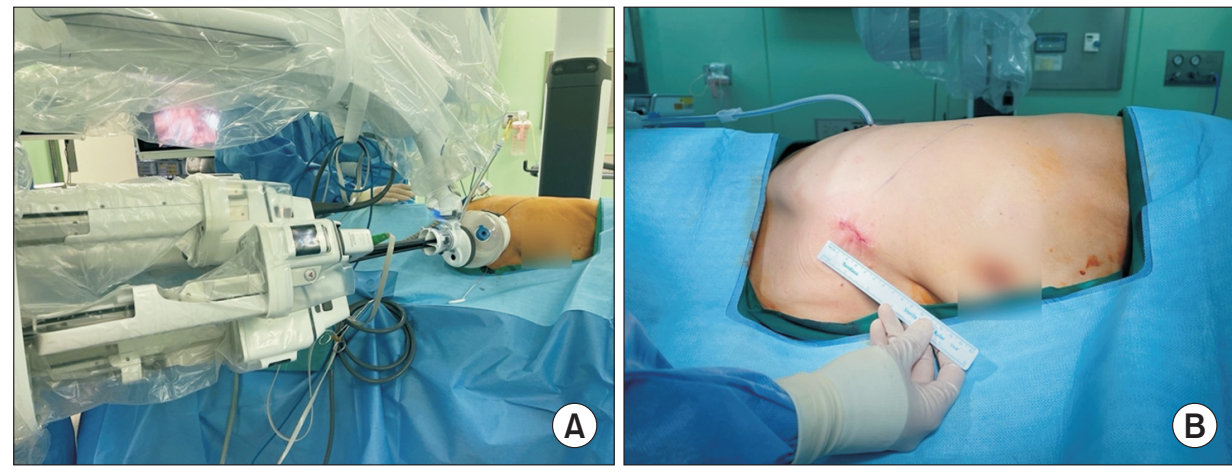

Fig. 2. (A) Subcostal incision with the patient in the semi-prone position. (B) A 3-cm-long postoperative wound. (A) Docking of the singleport system via the subcostal approach. (B) Wound after the operation. Written informed consent for publication of this image was obtained from the patient.

incision was then made right below the subcostal margin at the mid-clavicular line and a tunnel was dissected superiorly with long Metzenbaum scissors and fingers. Connection of the tunnel from the subcostal skin incision into the thoracic cavity was confirmed with an endoscope through the $5-\mathrm{mm}$ port. The wound retractor of the port access system was placed through the skin incision, and the robotic single-port (SP) surgical system (da Vinci SP Surgical System, Model SP1098; Intuitive Surgical Inc., Sunnyvale, CA, USA) was docked through the port access system (Fig. 2A). The dissection of the esophagus began at the lower esophagus and continued to the upper esophagus. The grasping power of the robotic instrument was insufficient to lift the esophagus; therefore, the esophagus was pushed or pulled using the instrument itself to secure space between the esophagus and pericardium or trachea, and the space was dissected using robotic monopolar or bipolar devices. During dissection of the upper mediastinum, an incidental injury of the thoracic duct occurred, but the thoracic duct was successfully ligated with a robotic clip applier. The robot docking time and console time for the esophagectomy were 10 and 41 minutes, respectively. Unedited surgical procedures are recorded in Supplementary Video 1. The skin incision was $3 \mathrm{~cm}$ (Fig. 2B). After esophagectomy, total laryngectomy was done by an otolaryngologist using the SP system, and a laryngo-gastrostomy was made after gastric mobilization using open laparotomy. The total operation time was 427 minutes. Because esophagectomy was done for the gastric pull-up, mediastinal lymph node dissection was not done in the thoracic cavity, and the lymph node at the upper mediastinum was dissected through a neck incision after laryngectomy. The numbers of dissected left and right recurrent laryngeal nerve lymph nodes were 2 and 5, respectively. A postoperative 1 week, the peak numerical pain intensity scale was 2 .

The pathologic report revealed that the main lesion was hypopharyngeal invasive squamous cell carcinoma (size:
$6.5 \times 3.5 \mathrm{~cm}$ ), invading the submucosa of upper esophagus and extending to the post-cricoid area of the hypopharynx. No metastatic lymph nodes were observed. During the postoperative periods, the patient suffered from anastomotic leakage, but it recovered in response to vacuum therapy. The author obtained verbal informed consent from the patient for publication of this case report and accompanying images.

\section{Discussion}

Despite advances in perioperative management, esophagectomy is one of the most invasive procedures among all surgical procedures for malignant tumors, with serious postoperative complications [1]. The morbidity and mortality rates have been reported to be up to $60 \%$ and $3.4 \%$, respectively, according to a large Japanese national report [2]. Therefore, minimally invasive esophagectomy can be an attractive alternative to conventional open esophagectomy for reducing postoperative morbidities and mortalities [3]. The TIME Trial (Time to Reduce Mortality in EndStage Renal Disease Trial), which was a phase III randomized controlled trial, compared thoracoscopic esophagectomy to open esophagectomy and reported that the incidence of pulmonary infection was considerably lower in the thoracoscopic esophagectomy group than in the open esophagectomy group [4]. In this context, many thoracic surgeons think that trauma to the thoracic cavity and its related morbidities could be reduced by decreasing the number of ports.

The SP system was approved in South Korea in the field of general thoracic surgery in South Korea. The SP system has 3 flexible instruments in contrast to the 2 non-flexible arms in the previous robotic single-site platform and a stereoscopic binocular camera, all contained within a $2.5-\mathrm{cm}-$ diameter cannula. Application of the SP system in esophagectomy has been attempted by Chiu et al. [5] in the 
cadaver model via a transcervical approach; the rationale of the transcervical approach is that the incidence of pneumonia remains significant, as transthoracic esophagectomy requires 1-lung ventilation. However, the anatomic landmarks in the transcervical approach are not familiar for thoracic surgeons, and other esophageal procedures such as excision of esophageal submucosal tumors cannot be done with the transcervical approach. In the previous cadaver study performed by the author [6], the subcostal approach in the semi-prone position could provide the appropriate surgical view for the posterior mediastinum and esophagus. In addition, rapid and appropriate management for emergent situations such as bleeding is possible in the subcostal approach. Even though entrance into the thoracic cavity could increase more pulmonary complications than the transcervical approach, the author believes that the subcostal approach is appropriate for esophageal procedures. To the author's knowledge, this case is the first esophagectomy using an SP system. As a pilot approach, the author performed esophageal mobilization in a patient with hypopharyngeal cancer with upper esophageal extension within 41 minutes. The chyle leakage from the thoracic duct in upper mediastinum was successfully ligated with a robotic clip applier during the operation. Even though the patient experienced anastomotic leakage, this was not related to the esophagectomy itself.

In conclusion, this case report shows that esophagectomy using an SP system via the subcostal approach is feasible and safe. More complex procedures such as mediastinal lymph node dissection could be attempted using SP systems in the future.

\section{Conflict of interest}

No potential conflict of interest relevant to this article was reported.

\section{ORCID}

Seong Yong Park: https://orcid.org/0000-0002-5180-3853

\section{Supplementary materials}

Supplementary materials can be found via https://doi. org/10.5090/jcs.21.102. Supplementary Video 1. Unedited video of robotic esophagectomy using single-port system.

\section{References}

1. Booka E, Takeuchi H, Nishi T, et al. The impact of postoperative complications on survivals after esophagectomy for esophageal cancer. Medicine (Baltimore) 2015;94:e1369.

2. Takeuchi H, Miyata H, Gotoh M, et al. A risk model for esophagectomy using data of 5354 patients included in a Japanese nationwide web-based database. Ann Surg 2014;260:259-66.

3. Lee JH, Hong JI, Kim HK. Robot-assisted thoracic surgery in nonsmall cell lung cancer. J Chest Surg 2021;54:266-78.

4. Biere SS, van Berge Henegouwen MI, Maas KW, et al. Minimally invasive versus open oesophagectomy for patients with oesophageal cancer: a multicentre, open-label, randomised controlled trial. Lancet 2012;379:1887-92.

5. Chiu PW, Ng SS, Au SK. Transcervical minimally invasive esophagectomy using da Vinci(R) SP(TM) Surgical System: a feasibility study in cadaveric model. Surg Endosc 2019;33:1683-86.

6. Park SY, Stein H, Heo SY. Preclinical, cadaveric study of the application of da Vinci single port system in thoracic surgery. J Thorac Dis 2019;11:5586-91. 